\title{
Editorial: Above-belowground interactions involving plants, microbes and insects
}

\author{
Ana Pineda ${ }^{1 *}$, Roxina Soler ${ }^{2,3}$, Maria J. Pozo ${ }^{4}$, Sergio Rasmann ${ }^{5}$ and Ted C. J. Turlings ${ }^{6}$ \\ 'Laboratory of Entomology, Wageningen University, Wageningen, Netherlands, ${ }^{2}$ Department of Terrestrial Ecology, \\ Netherlands Institute of Ecology (NIOO-KNAW), Wageningen, Netherlands, ${ }^{3} R \& D$ Microbiology, Koppert Biological Systems, \\ Berkel en Rodenrijs, Netherlands, ${ }^{4}$ Department of Soil Microbiology and Symbiotic Systems, Estación Experimental del \\ Zaidin, CSIC, Granada, Spain, ${ }^{5}$ Laboratory of Functional Ecology, Institute of Biology, University of Neuchâtel, Neuchâtel, \\ Switzerland, ${ }^{6}$ Laboratory of Fundamental and Applied Research in Chemical Ecology, Institute of Biology, University of \\ Neuchâtel, Neuchâtel, Switzerland
}

Keywords: plant defenses, plant-insect interactions, beneficial microbes, root herbivores, signaling pathways, multitrophic interactions, phytohormones, induced resistance

"Integration" has become a central theme in plant research, and this concept has greatly contributed to the exciting recent advances of our understanding of plant interactions with their environment. Plants in nature interact with a plethora of organisms, from microbes and insects through vertebrates to other plants. While some organisms are detrimental, others have mutualistic interactions with plants. Whereas, plant responses to all these organisms were initially studied separately for aboveground and for belowground domains, nowadays it is accepted that plant interactions belowground orchestrate a cascade of events that affects the interactions of plants with organisms that live aboveground, and vice versa. In parallel, it has become evident that the plants' interactions with both mutualists and antagonists are governed by often similar phytohormonal and metabolic responses, which starting at the molecular level, shape the ecological interactions that take place in natural and agricultural systems. Beyond the fundamental interest in these interactions, a comprehensive understanding of how plants integrate their responses to microbes and insects below-aboveground, will also contribute to tackle applied concerns such as the development of sustainable crop protection strategies, and the development of novel crop varieties for increasing yield and performance. Integrating plant responses to beneficial and detrimental organisms, from microbes to insects, and using integrative approaches that range from the molecular changes within the plant to the ecological interactions taking place among organisms, will provide a more representative view of the multitude and complexity of interactions in nature. With this Research Topic, we aimed at being integrative, by proposing a wide array of themes, spanning papers dealing with the molecular bases of above-belowground plant-insect-microbe interactions, to more ecological and large-scale work.

The integration of several approaches to study belowground interactions is the focus of a significant methodological contribution of Campos-Herrera et al. (2013), where the authors review recent advances in molecular, chemical, and spatial techniques for studying belowground ecology. Particularly, they expand on how molecular tools could be developed and used to measure diversity and distribution of soil nematodes, and propose how can it be exploited in biological control. Nonetheless, the steep road necessary to uncover mechanisms of interaction happening in the soil has just started, and these authors nicely outline how collaboration with different fields will be continue to be necessary in future research belowground. In a different framework, integration of below-aboveground research is what prompted Balmer and Mauch-Mani (2013) to pose the intriguing question of why plant organs such as leaves and roots mount such different defense responses against their microbial and herbivorous attackers. They concluded that roots and shoots 
use different defense machinery, and using an evolutionary and ecological approach, this could be explained by feedback mechanisms between plants and the microbial communities.

It is estimated that all plant species establish symbioses with microbes. Most of these so-called beneficial microbes are located belowground in the rhizosphere, where vast numbers of different microbial species reside. The plants as we perceive them aboveground are the result of millions of years of coevolution with these beneficial microbes. From this perspective, Van Geem et al. (2013) discuss how above-belowground interactions across several trophic levels may have been driving genetic variation in plant traits. As a consequence, plant-insect and plantpathogen interactions aboveground would have been indirectly (and secretly) shaped by symbiotic associations belowground. Focusing on beneficial microbes belowground, Pangesti et al. (2013) discuss the how moving from addressing interactions between plants and single microbial species to a community approach could move forward our understanding of the complex soil ecosystem. Their major take-home message is that following this community approach below and aboveground in combination with the understanding of induced systemic resistance (ISR), will increase the reliability and durability of the application of beneficial microbes. A long-standing question in the studies of microbes and insects is: what is driving the context-dependency of the effects of beneficial microbes on insects? Barber et al. (2013) provide an interesting example where different mycorrhizal strains impact both herbivores and pollinators in a way that depends both on the mycorrhizal fungi and insect identity. Vannette et al. (2013) move a step further, and show that plant genotype can also be a crucial factor in these interactions. They point out that the effects of mycorrhizal fungi on, for instance, cardenolides, the main defensive compounds of milkweeds (Asclepia spp.), vary with plant species. Most fascinating is the fact that this seems to be explained by the phylogenetic origin of those species, and it appears that the tradeoff between plant growth and defense can be mitigated by mycorrhizal inoculation. Working with a different group of soildwelling organisms, Wurst (2013) explores the underappreciated role of the indirect linkage between detritivores and plants in plant-mediated above-belowground interactions. Indeed, even though detritivorous soil fauna is ubiquitous and present in high abundances, it is still highly underrepresented in abovebelowground studies. The few existing studies in this regard highlight both positive and negative effect of detritivores on plant resistance, thus currently impairing generalizations on the topic.

Phytohormones, and particularly jasmonic acid (JA) and salicylic acid (SA), have been shown to orchestrate induced plant defenses to herbivory and pathogen infection. Nevertheless, how plants integrate their responses when under multiple belowaboveground attackers is not yet fully understood. For instance, the role of JA signaling goes beyond effects on herbivores, and with their experimental research Constantino et al. (2013) highlight the importance of belowground ISR on aboveground tissues. In a detailed experimental paper, they show that the JA-biosynthetic gene LOX3 of Zea mays is a negative regulator of ISR, and down-regulation of its expression in the roots by the beneficial soil fungi Trichoderma virens is required for triggering ISR on the leaves. Interesting is also their finding that the xylem sap contained the responsible factor for these effects. Along the same lines, Shavit et al. (2013) showed how JA and SA signaling pathways may mediate the positive effects of plant growth-promoting rhizobacteria (PGPR) on whiteflies aboveground. They observed a decrease of the induction of JA and SA-marker genes by PGPR, and propose that changes in the microbial community of the rhizosphere may trigger this effect.

Other hormones, which are traditionally considered to be involved in plant growth and development, are also gaining attention as possible regulators of plant interactions with microbes and insects (Pangesti et al., 2013). These hormones include auxines, cytokinines, or abscisic acid (ABA), with the latter being known for its crucial role in responses to abiotic stress. In a comprehensive experimental paper, Vos et al. (2013) show that ABA is an important regulator of herbivoreinduced plant resistance. More specifically, a functional ABA signaling pathway is required to mediate the effect of previous herbivory on the reduction of subsequent herbivore performance and the associated induction of JA-regulated responses. These studies substantiate the fact that plant signaling pathways can regulate responses to all kinds of stresses, from abiotic to biotic, from microbes to insects, and from mutualistic to detrimental interactions.

One major concern this decade is facing, is how climate change may alter plant-mediated interactions among root and shoot associated organisms. Little is known, and almost no empirical evidence has been generated on this matter. In a perspective article McKenzie et al. (2013) explore this relevant issue, highlighting the importance of the belowground domain when attempting predictions on the effects of climate change on plant-insect interactions aboveground. Providing rare experimental evidence, Ryalls et al. (2013) show how elevation of temperatures and $\mathrm{CO}_{2}$ can influence belowaboveground interactions between plants, microbes, and insects. Specifically, they show that elevation of temperatures and $\mathrm{CO}_{2}$ had no effects on aphids, whereas had contrasting effects on weevils that potentially occurred through changes in root nodulation patterns. By predicting plant species distributions along environmental gradients, Pellissier et al. (2013) explore the long-standing question of what drives plant species assemblages in space. The underlying hypothesis of their work is that plant and fungi mutually influence their spatial distribution. They show that by including information on fungal abundance and identity, it is possible to significantly improve the predictive accuracy of plant species distribution models, especially for plant species occurring at high elevations. Indeed, colonization of high elevation soils should be facilitated by the presence of mycorrhizal fungi, but this awaits experimental confirmation.

One another major contemporary challenge in plant research is how to achieve an economically, sustainably, and environmentally-sound increase in the production of high quality foods. Fundamental knowledge of how plants interact with their attackers and beneficials provides the basis of modern agriculture. An example of this is presented in the experimental article by Pierre et al. (2013), in which it is shown how the application of the phytohormones SA and JA on broccoli plants 
decreases the incidence of several devastating insects pests, both below- and aboveground. Interestingly, these effects were not mediated by glucosinolate induction, and they only affected specialist herbivores and not generalists, a rather uncommon pattern that merits future attention.

A fascinating and very promising topic, but still scarce in the literature, is the fact the some beneficial rhizobacteria also have a direct antagonistic effect on root herbivores. The review by Kupferschmied et al. (2013) provides a state of the art of this topic. Insecticidal properties of Pseudomonas bacteria are proposed as a novel biological tool to tackle important root herbivore pests, with the advantage of a high persistence due to their adaptation to the life on plant roots. Along the same lines but in a wider context, Orrell and Bennett (2013) take on the challenging question of how fundamental knowledge on plant-mediated above-belowground interaction can be exploited in practice to enhance crop productivity. They suggest that the manipulation of above-belowground interactions may help to reduce losses to pests and increase crop yield by either implementing better intercropping strategies, by selecting for specific plant traits that mediate beneficial above-belowground interactions, or by breeding mutualistic organisms to enhance the benefits of the interactions.

In this Research Topic we aimed at highlighting the need for a good understanding of plant-mediated below-aboveground

\section{References}

Balmer, D., and Mauch-Mani, B. (2013). More beneath the surface? Root versus shoot antifungal plant defenses. Front. Plant Sci. 4:256. doi: 10.3389/fpls.2013.00256

Barber, N. A., Kiers, E. T., Hazzard, R. V., and Adler, L. S. (2013). Contextdependency of arbuscular mycorrhizal fungi on plant-insect interactions in an agroecosystem. Front. Plant Sci. 4:338. doi: 10.3389/fpls.2013.00338

Campos-Herrera, R., Ali, J. G., Diaz, B. M., and Duncan, L. W. (2013). Analyzing spatial patterns linked to the ecology of herbivores and their natural enemies in the soil. Front. Plant Sci. 4:378. doi: 10.3389/fpls.2013.00378

Constantino, N., Mastouri, F., Damarwinasis, R., Borrego, E., Moran-Diez, M. E., Kenerley, C. M., et al. (2013). Root-expressed maize lipoxygenase 3 negatively regulates induced systemic resistance to Colletotrichum graminicola in shoots. Front. Plant Sci. 4:510. doi: 10.3389/fpls.2013.00510

Kupferschmied, P., Maurhofer, M., and Keel, C. (2013). Promise for plant pest control: root-associated pseudomonads with insecticidal activities. Front. Plant Sci. 4:287. doi: $10.3389 /$ fpls.2013.00287

McKenzie, S. W., Hentley, W. T., Hails, R. S., Jones, T. H., Vanbergen, A. J., and Johnson, S. N. (2013). Global climate change and above- belowground insect herbivore interactions. Front. Plant Sci. 4:412. doi: 10.3389/fpls.2013.00412

Orrell, P., and Bennett, A. E. (2013). How can we exploit above-belowground interactions to assist in addressing the challenges of food security? Front. Plant Sci. 4:432. doi: 10.3389/fpls.2013.00432

Pangesti, N., Pineda, A., Pieterse, C. M. J., Dicke, M., and Van Loon, J. J. A. (2013). Two-way plant mediated interactions between root-associated microbes and insects: from ecology to mechanisms. Front. Plant Sci. 4:414. doi: 10.3389/fpls.2013.00414

Pellissier, L., Pinto, E., Niculita-Hirzel, H., Moora, M., Villard, L., Goudet, J., et al. (2013). Plant species distribution along environmental gradient: do belowground interactions with fungi matter? Front. Plant Sci. 4:500. doi: 10.3389/fpls.2013.00500

Pierre, S. P., Dugravot, S., Hervé, M. R., Hasan, H., Van Dam, N. M., and Cortesero, A. M. (2013). Belowground induction by Delia radicum or phytohormones interactions between insects and other organisms, and that this requires the integration of both mechanistic and more holistic, ecological approaches. This diverse set of excellent contributions has also the merit to highlight that nature as we observe it aboveground, is the product of a diffuse coevolutionary history between plants and a multitude of other organisms, living above- and belowground. It is therefore not surprising that roots are now being considered the key of the second green revolution, and the study of their interactions with organisms living in the rhizosphere should be now fully integrated to boost food production in a sustainable manner.

\section{Acknowledgments}

We would like to thank all the authors and reviewers. Their excellent input and creativity have made this research topic on below-aboveground interactions possible. Research activities of the Topic editors are supported by several funding agencies: AP by the Netherlands Organization for Scientific Research (NWO) [ALW grant No. 822.01.005], MP by the Spanish Ministry of Economy and Competitiveness (AGL201239923), SR by a National Science Foundation Ambizione grant PZ00P3_131956/1, and TT by the Swiss National Science Foundation (economic stimulus grant).

affect aboveground herbivore communities on field-grown broccoli. Front. Plant Sci. 4:305. doi: 10.3389/fpls.2013.00305

Ryalls, J. M. W., Riegler, M., Moore, B. D., Lopaticki, G., and Johnson, S. N. (2013). Effects of elevated temperature and CO2 on aboveground-belowground systems: a case study with plants, their mutualistic bacteria and root/shoot herbivores. Front. Plant Sci. 4:445. doi: 10.3389/fpls.2013.00445

Shavit, R., Ofek-Lalzar, M., Burdman, S., and Morin, S. (2013). Inoculation of tomato plants with rhizobacteria enhances the performance of the phloem-feeding insect Bemisia tabaci. Front. Plant Sci. 4:306. doi: 10.3389/fpls.2013.00306

Van Geem, M., Gols, R., Van Dam, N. M., Van Der Putten, W. H., Fortuna, T., and Harvey, J. A. (2013). The importance of aboveground-belowground interactions on the evolution and maintenance of variation in plant defence traits. Front. Plant Sci. 4:431. doi: 10.3389/fpls.2013.00431

Vannette, R. L., Hunter, M. D., and Rasmann, S. (2013). Arbuscular mycorrhizal fungi alter above- and below-ground chemical defense expression differentially among Asclepias species. Front. Plant Sci. 4:361. doi: 10.3389/fpls.2013.00361

Vos, I. A., Verhage, A., Schuurink, R. C., Watt, L. G., Pieterse, C. M. J., and Van Wees, S. C. M. (2013). Onset of herbivore-induced resistance in systemic tissue primed for jasmonate-dependent defenses is activated by abscisic acid. Front. Plant Sci. 4:539. doi: 10.3389/fpls.2013.00539

Wurst, S. (2013). Plant-mediated links between detritivores and aboveground herbivores. Front. Plant Sci. 4:380. doi: 10.3389/fpls.2013.00380

Conflict of Interest Statement: The authors declare that the research was conducted in the absence of any commercial or financial relationships that could be construed as a potential conflict of interest.

Copyright (C) 2015 Pineda, Soler, Pozo, Rasmann and Turlings. This is an open-access article distributed under the terms of the Creative Commons Attribution License (CC $B Y)$. The use, distribution or reproduction in other forums is permitted, provided the original author(s) or licensor are credited and that the original publication in this journal is cited, in accordance with accepted academic practice. No use, distribution or reproduction is permitted which does not comply with these terms. 